\title{
Imaging Biomineralization in Flow Systems
}

\author{
Logan Schultz,* Betsey Pitts, ${ }^{*}$ Robin Gerlach*
}

*Center for Biofilm Engineering, Montana State University, 366 EPS, Bozeman, MT, 59717

The precipitation and stability of minerals is strongly correlated with the local chemical environment. The significant influence of bacteria and other micro-organisms on local chemistry in flow scenarios can lead to biologically-induced mineral precipitation. Microscopy can be utilized to characterize the spatial and temporal development of microbes, minerals, and extracellular polymeric substances (EPS) in a fully hydrated and non-invasive manner. Due to a lack of dehydration artifacts, this imaging situation is tremendously useful for elucidating spatial relationships of these components.

In this study, the soil bacterium Sporosarcina pasteurii was introduced in flow cells with $1 \mathrm{~mm}$ pore spaces and grown in the presence of a medium containing dissolved calcium and urea. $S$. pasteurii effectively hydrolyzes urea, which increases the $\mathrm{pH}$ and induces calcite precipitation. Stereo microscopy conducted in real-time demonstrated temporal and spatial differences in precipitation. Analysis of these images quantified precipitate size and density, providing a basis for calculations in pore-space reduction and substrate conversion.

Confocal scanning laser microscopy after staining with Syto11 and C2-dichlorotriazine was used to image the spatial relationships of the cells, EPS, and minerals. It was expected that the Syto 11 would bind to nucleic acids associated with cells and the C2-dichlorotriazine would bind to alcohols and polysaccharides associated with EPS. While maintaining a fully hydrated state in the reactors, two-dimensional images were taken at $0.5 \mu \mathrm{m}$ depth increments through the samples and compiled in z-stacks to reproduce three-dimensional topographical images. It was evident from the images that the bacteria adhered preferentially to the calcite surfaces. Ongoing investigations are seeking to gain a better understanding of the association of bacteria throughout the developmental stages of the precipitates and better understand the biological environment at the interior of the precipitates.

In designing methods to control biomineralization in fouling scenarios or to manipulate hydraulic transport properties of porous media, novel methods in microscopy prove to be an invaluable tool. This presentation will highlight some techniques, capabilities, and difficulties associated with imaging biomineralization in flow systems. 


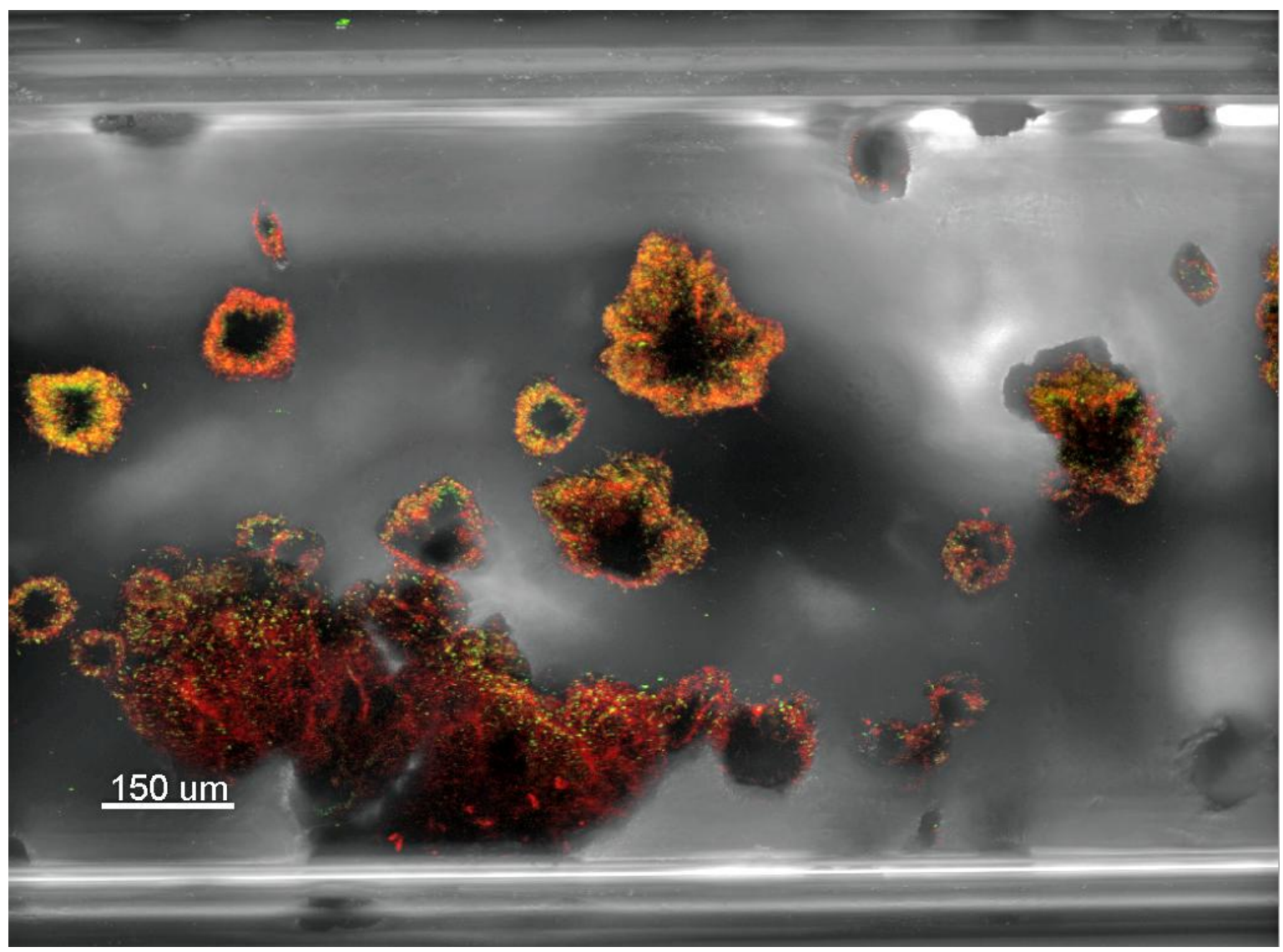

Figure 1. Confocal scanning microscope image of a capillary flow cell with biomineralized calcite surrounded by fluorescence stained microbial communities.

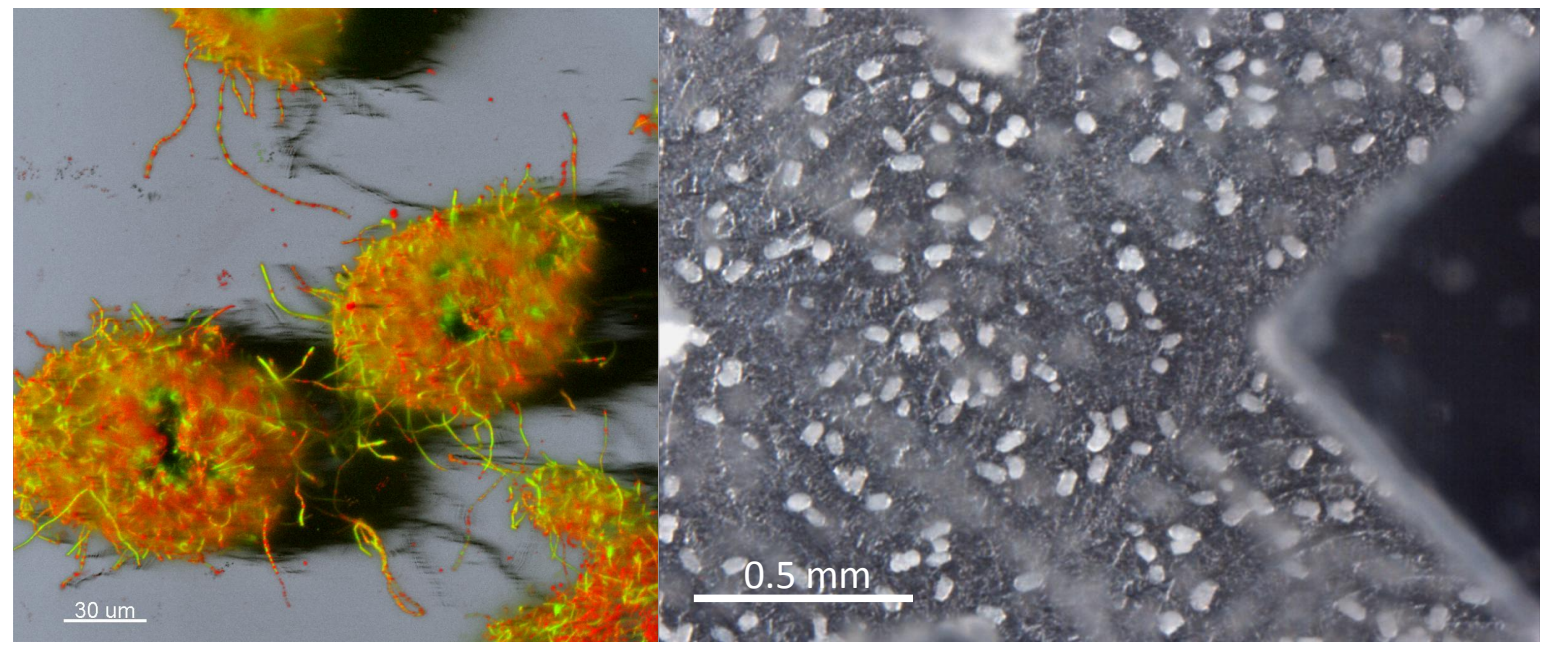

Figure 2. (left) Three-dimensional reconstruction of surface attached calcite, elucidating the prolific attachment of microbes to the calcite exterior. (right) Stereo microscope image of calcite attached to the bottom of a flow cell, providing the means to characterize size and distribution of precipitates. 